Research Article

\title{
Electromagnetic Simulation of the Near-Field Distribution around a Wind Farm
}

\author{
Shang-Te Yang and Hao Ling \\ Department of Electrical and Computer Engineering, The University of Texas at Austin, Austin, TX 78712, USA \\ Correspondence should be addressed to Shang-Te Yang; shangte.yang@utexas.edu
}

Received 13 October 2012; Revised 31 December 2012; Accepted 10 January 2013

Academic Editor: David de la Vega

Copyright (C) 2013 S.-T. Yang and H. Ling. This is an open access article distributed under the Creative Commons Attribution License, which permits unrestricted use, distribution, and reproduction in any medium, provided the original work is properly cited.

\begin{abstract}
An efficient approach to compute the near-field distribution around and within a wind farm under plane wave excitation is proposed. To make the problem computationally tractable, several simplifying assumptions are made based on the geometry problem. By comparing the approximations against full-wave simulations at $500 \mathrm{MHz}$, it is shown that the assumptions do not introduce significant errors into the resulting near-field distribution. The near fields around a $3 \times 3$ wind farm are computed using the developed methodology at $150 \mathrm{MHz}, 500 \mathrm{MHz}$, and $3 \mathrm{GHz}$. Both the multipath interference patterns and the forward shadows are predicted by the proposed method.
\end{abstract}

\section{Introduction}

Due to the rapid development of wind energy around the globe, the adverse effect of large wind turbines on radar and communication systems is raising serious concerns [17]. Both theoretical and experimental studies have been conducted to assess the impact of wind turbines on various systems including weather radar [1], air traffic control radar [2], air defense radar [3,4], and marine radar [5], as well as radio communication and navigation systems [6,7]. Two types of electromagnetic phenomenology can give rise to interference effects : radar clutter and electromagnetic transmission interference. Wind turbine clutter arises from the backscattered signal from the turbine structures and is of concern mainly to radar systems. Due to the spinning turbine blades, the resulting Doppler clutter can interfere with the detection of moving targets such as weather and aircraft. This topic has been well studied to date. For example, the United States Air Force Research Laboratory collected in situ measurement data of several utility class wind turbines in Fenner, NY, USA and corroborated the measurements with high-frequency ray-tracing simulation $[8,9]$. The detailed phenomenology of the backscattering from wind turbines is fairly well understood.
The issue of electromagnetic transmission interference, on the other hand, is more subtle and therefore more difficult to assess. There exists a shadow region directly behind a turbine structure where the scattered electromagnetic field cancels destructively with the incident electromagnetic field. Outside the shadow region, the scattered field and incident field interfere due to their phase difference, forming a multipath region characterized by a rapid spatial oscillation pattern [10]. If a receiver in a communication link is positioned sufficiently close to a wind turbine (or a cluster of turbines), the receiver could experience signal fades. Similarly for a radar system, if a target of interest comes sufficiently close to a wind farm, the received radar signal from the target will experience a two-way signal transmission loss through the wind farm. The aforementioned effects are strongly dependent on the locations of the transmitter and receiver with respect to the wind turbine structure. Therefore, this issue also deserves careful consideration.

Both theoretical and experimental works have been conducted on the characterization of the transmission interference problem. A simple, approximate geometrical blockage estimate can be derived based on the Fresnel zone argument [11]. This is the standard methodology used to estimate 
the shadowing effect due to wind turbine structures by the United States Federal Aviation Administration obstruction evaluation process $[12,13]$. The radar cross section (RCS) concept was applied to address the multipath interference problem in [14] and to estimate the loss of radar detection range in [15]. Full-wave analysis was used to analyze the shadow due to a turbine tower under a pencil beam excitation in [16]. Measurements of the shadow behind a wind turbine were carried out in $[17,18]$. The effect of an offshore wind farm on radio communication and navigation systems was measured in-situ in [6]. The wind farm transmission interference problem has received less attention thus far in comparison to the radar clutter issue. This may be due to several reasons. From the measurement perspective, the transmission problem involves a bistatic transmitter/receiver configuration, which is logistically more difficult to carry out. From the computational perspective, near-field simulation around an entire wind farm poses a large computational burden. As a result, the interference effect of an entire wind farm on signal transmission is not yet well characterized.

In this paper, we set out to develop an efficient but approximate electromagnetic approach to compute the received field strength within and around a wind farm. Our approach is based on several simplifying assumptions. First, the turbine scattering is assumed to be dominated by the tower structure of the turbine, while additional scattering from the blades and nacelle structures are assumed to be of secondary importance. Second, due to the large length-tocross section ratio of typical tower structures, the scattering process is assumed to be predominately two-dimensional (2D) in nature for an observer located close to the turbine. Third, we assume that the scattered fields can be predicted by using the far-field complex echo width (EW) of the tower. Lastly, the individual turbines in a wind farm are assumed to be excited under the incident excitation while neglecting the interaction terms. This is also known as the Born approximation. Using these assumptions, we formulate an approximate prediction methodology that leads to a significant reduction in the computational complexity of the problem and allows us to predict the shadow and multipath interference within and around a large wind farm.

This paper is organized as follows. In Section 2, we establish the validity of the three assumptions that form the basis of our prediction methodology. Full-wave simulation based on the multilevel fast multipole method (MLFMM) in the commercial electromagnetic package FEKO [19] is used to check the validity of the assumptions. In Section 3, we apply our prediction approach to two or more turbines by ignoring the interturbine interactions. It is again shown by comparison with full-wave simulation that such approach leads to a reasonable approximation barring any occlusion effects among the turbines. Results for a threeby-three farm are then shown, and the behavior of the fields as a function of frequency is investigated. The savings in computation time are also summarized and discussed in Section 3. Discussions and conclusions are given in Section 4.

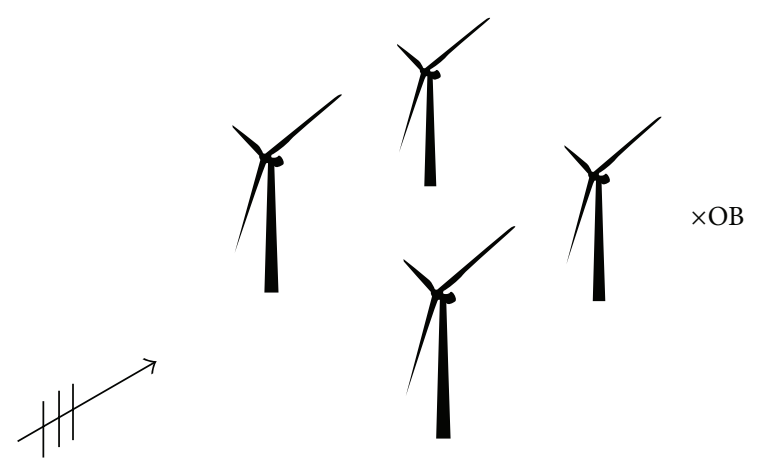

FIGURE 1: Illustration of the problem statement.

\section{EM Simulation Methodology: Single-Turbine Case}

The problem of interest is illustrated in Figure 1. The transmitter is assumed to be located far away, so that the incident field is a plane wave. The field at an arbitrary observation point $O B$ in close proximity of the wind farm is desired. Of particular interest to us is the case where both the transmitter and the receiver are located along the same horizontal plane, as our research is motivated by the impact on marine radar and communication systems from offshore wind farms. The more general configuration of arbitrary transmitter and receiver elevation angles, although interesting, is outside the scope of the present work. Clearly, a full-wave solution to this problem at frequencies above $1 \mathrm{GHz}$ is computationally too exhaustive to carry out even using MLFMM, which already requires much less memory and time than the traditional method of moments (MoM). Here, we describe several simplifying approximations to arrive at the desired field distribution for a single turbine. In the next section, the Born approximation will be further applied to deal with multiple turbines that comprise a farm.

2.1. Geometry Simplification. While a turbine consists of multiple components including the tower, the blades, and the nacelle, it is believed that the tower gives rise to the strongest scattering and shadowing effects. Angulo et al. simulated the RCS of individual components of a turbine using physical optics (PO) and showed that the tower gives the strongest contribution [20]. To test the validity of this assumption under a near-field observation, we first simulate a single turbine at $500 \mathrm{MHz}$ and compute the electric field distribution around the structure. The turbine model consists of a long cone-shaped tower, a rectangular-shaped nacelle, and turbine blades which are modeled by thin plates. The tower is $64.5 \mathrm{~m}$ tall with a $3.8 \mathrm{~m}$ bottom diameter and $2.8 \mathrm{~m}$ top diameter. The nacelle is $15 \mathrm{~m}$ long and has a $3 \mathrm{~m} \times 3 \mathrm{~m}$ cross section. The three turbine blades are modeled as $34.3 \mathrm{~m}$ long and $3 \mathrm{~m}$ wide plates with a $15^{\circ}$ pitch angle with respect to the rotation plane. All three components are assumed to be perfect conductors for simplicity, although different materials could be modeled without too much additional effort by using an impedance boundary condition approximation. 
The turbine is excited with a plane wave at zero elevation and $30^{\circ}$ with respect to hub-on incidence in azimuth. The incident wave is vertically polarized with an electric field strength of $1 \mathrm{~V} / \mathrm{m}$. The full-wave simulation is carried out using the MLFMM solver in FEKO. The resulting total electric field in the vertical polarization is plotted at a height of $z=34.25 \mathrm{~m}$ with a sampling interval of $5 \mathrm{~m}$ in the $x y$ plane in Figure 2(a). The turbine is located at $x=y=0$. The field strength is plotted in $\mathrm{dB}$ with respect to $1 \mathrm{~V} / \mathrm{m}$, and the dynamic range is $10 \mathrm{~dB}$. A shadow region behind the turbine is clearly observed. The shadow is the darkest immediately behind the turbine but gradually disappears as the distance increases. Outside the shadow region, rapidly oscillating interference patterns are observed. It should be noted that the sampling interval of $5 \mathrm{~m}$ does not fully capture the fine oscillation of the field pattern at $500 \mathrm{MHz}$.

To test the modeling fidelity required of the turbine structure, we simulate only the cone-shaped tower using MLFMM and plot the near-field distribution in Figure 2(b). Comparing Figure 2(b) to Figure 2(a), it is noted that the main difference is in the region at the bottom of the figures. In addition, the shadow region in Figure 2(a) has more fluctuation as compared to that in Figure 2(b), which may be due to the contribution from the nacelle on the top of the tower. To provide a more quantitative measure, the error between the two plots is computed. We define the percentage RMS error as

$$
\% \text { of RMS error }=\sqrt{\frac{\sum_{\text {pixels }}\left|I_{1}-I_{0}\right|^{2}}{\sum_{\text {pixels }}\left|I_{0}\right|^{2}}} \times 100 \%,
$$

where $I_{0}$ and $I_{1}$ are, respectively, the field magnitude of each pixel in the two figures on a linear scale. The RMS error between Figures 2(a) and 2(b) is 3.34\%. This result confirms that most of the near-field oscillations and the forward shadow region are contributed by the tower structure.

Next, we simulate the total field around a $64.5 \mathrm{~m}$ tall perfect conducting cylinder with the mean diameter of the cone-shaped tower, $3.3 \mathrm{~m}$, at $500 \mathrm{MHz}$ using FEKO. The field in the same observation plane is computed and plotted in Figure 3(a). Comparing Figure 3(a) to 2(b), it is observed that the two figures bear the same interference patterns across most of the regions of the plot. The main difference is again in the backscattering region. The RMS error between Figure 2(b) and Figure 3(a) is $3.42 \%$. This result indicates that the cone-shaped tower can be modeled by a finite cylinder with an acceptable error. Lastly, the RMS error between Figures 2(a) and 3(a) is $4.35 \%$. Therefore, the features of the turbine scattering can be mostly captured by that from a simplified tower alone.

2.2. $2 D$ Modeling. Since a simple cylinder can model the tower structure with acceptable error and since the cylinder is very long compared to its cross section, we next investigate the use of a 2D simulation to model the same problem. This amounts to using an infinitely long cylinder to model the finite one, neglecting the effect of the end truncation, and in the process turning the problem into a $2 \mathrm{D}$ one. To carry out the $2 \mathrm{D}$ simulation in FEKO, we use a short (a half wavelength in height) cylinder with a $3.3 \mathrm{~m}$ diameter bounded by periodic boundary conditions (PBCs) on the top and the bottom. The full-wave simulation is done using the MoM solver. This setup is equivalent to an infinitely long cylinder excited by a plane wave. The observation plane is at the center of the short cylinder, and the result is plotted in Figure 3(b). The interference pattern and strength are almost identical to those of the 3D finite cylinder. The only noticeable difference is that the shadow gradually fills out without the fluctuation observed in Figure 3(a), which is due to the two ends of the finite cylinder. The RMS error between the $3 \mathrm{D}$ full model MLFMM simulation and the $2 \mathrm{D}$ simulation (i.e., Figures $2(\mathrm{a})$ and $3(\mathrm{~b})$ ) is $4.38 \%$. The RMS error between the $3 \mathrm{D}$ finite cylinder simulation and the $2 \mathrm{D}$ simulation (Figures 3(a) and 3(b)) is only $0.09 \%$. The $2 \mathrm{D}$ approach dramatically reduces the number of unknowns and accelerates the simulation time. Detailed simulation time savings will be reported later in Section 3.

The previously mentioned approximation is valid because the finite cylinder is very long compared to its cross section, and we only observe the field at a very close distance. To see the maximum range for the validity of the $2 \mathrm{D}$ approximation, the following investigation is carried out. We study the radiation from a uniform, finite line current of length $64.5 \mathrm{~m}$. The result from numerical integration is shown as the solid blue curve in Figure 4. The figure is plotted on a log-log scale, and the curve shows a very clear break in slope at about $7000 \mathrm{~m}$. The break in slope suggests that the field changes from a $2 \mathrm{D}(1 / \sqrt{r})$ decay to a $3 \mathrm{D}(1 / r)$ decay. Also plotted in the dotted red line is the field due to an infinitely long line current [21]:

$$
E_{z}=-I \frac{\beta^{2}}{4 \omega \varepsilon} H_{0}^{(2)}(\beta r) \stackrel{\beta r \gg 0.25}{\approx}-\eta I \sqrt{\frac{2 j}{\pi \beta r}} e^{-j \beta r},
$$

where $I$ is the current strength, $\beta$ is the free space wave number, $\omega$ is the angular frequency, $\varepsilon$ is the permittivity of free space, $H_{0}^{(2)}$ is the Hankel function of the second kind with order zero, $r$ is the observation distance, and $\eta$ is the wave impedance of free space. It is seen that the field from the finite line current tracks this line quite well until the break in slope occurs. This implies that the $2 \mathrm{D}$ scattering model is a valid approximation for the field of a finite line current within a certain observation distance. Plotted in the dashed black line is the far field due to an infinitesimal-length point current given by

$$
E_{\theta}^{F F}=-\frac{j \beta \eta}{4 \pi r} I l e^{-j \beta r} \sin \theta
$$

where $I$ is the current strength, $l$ is the length of the current, and $\theta$ is the angle between the position vector of the observer and the direction of the current. In Figure $4, \theta$ is set to $90^{\circ}$. It is seen that the field of a finite-length current tracks this line after the break in slope. The distance at which the break in slope occurs can therefore be determined by equating (2) and (3) and solving for $r$. The result is $l^{2} / \lambda$ (or $6,939 \mathrm{~m}$ for the case under consideration). Note that this expression is different 


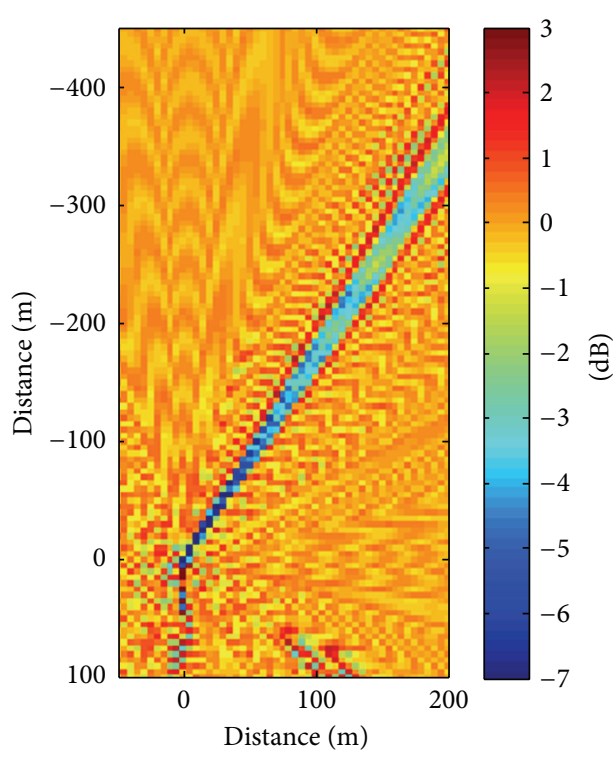

(a) 3D turbine model

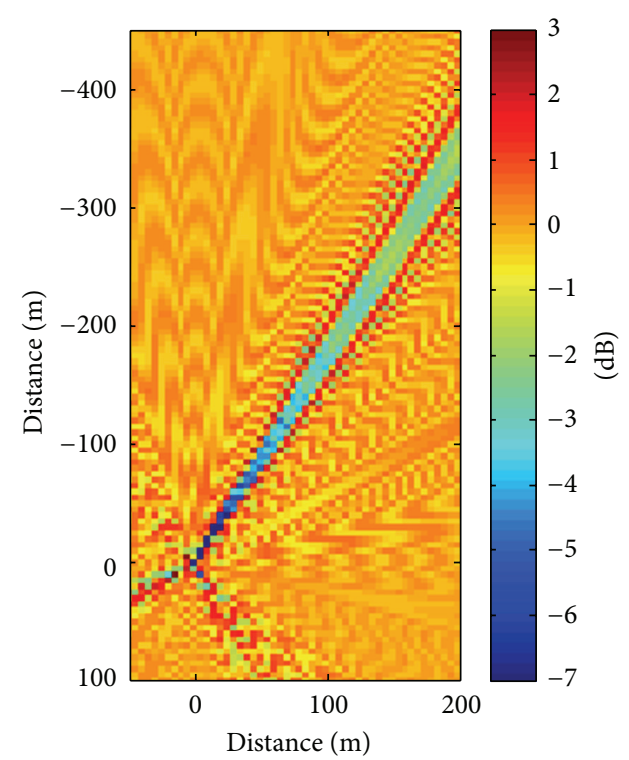

(b) 3D finite tower

FIGURE 2: Results of the single-turbine simulation at $500 \mathrm{MHz}$. (a) Near-field distribution around a 3D turbine model including the tower, nacelle, and blades. (b) Near-field distribution around the cone-shaped tower.

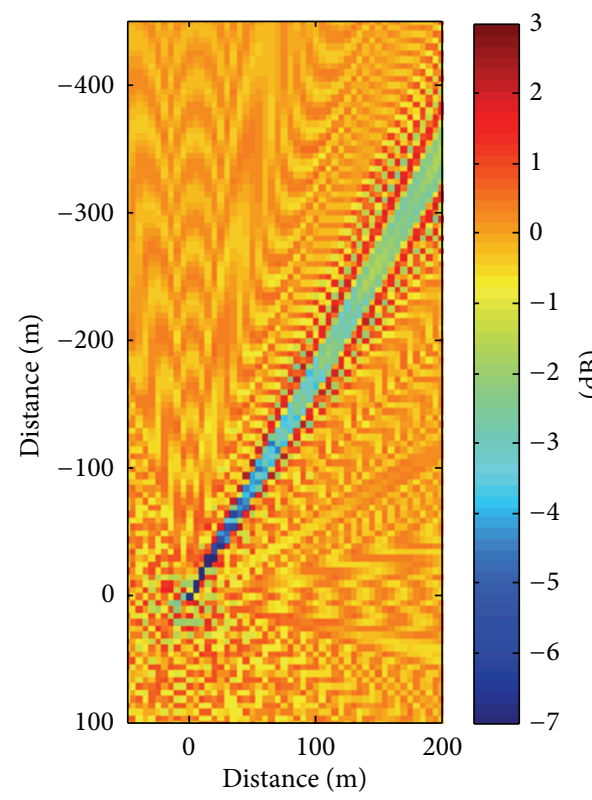

(a) 3D finite cylinder

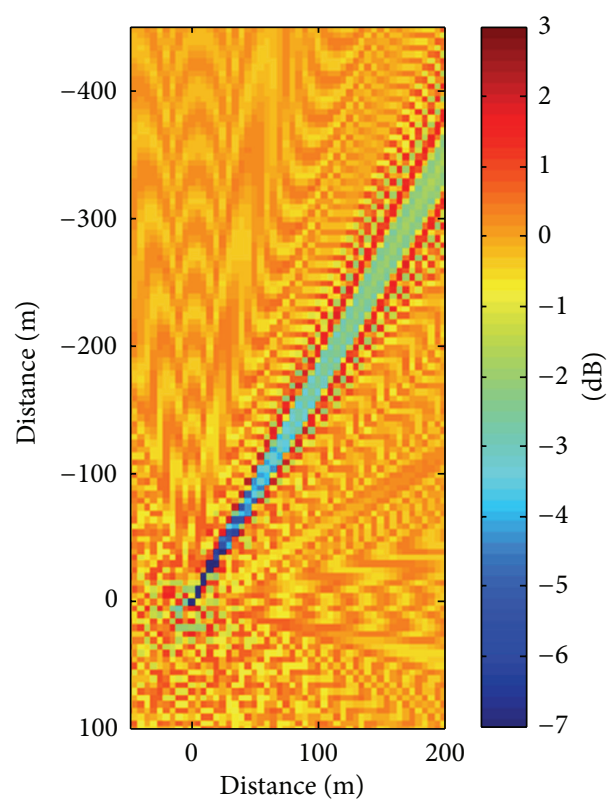

(b) infinite 2D cylinder

FIGURE 3: Results of the single-turbine simulation at $500 \mathrm{MHz}$. (a) Near-field distribution around a 3D finite cylinder. (b) Near-field distribution around an infinite 2D cylinder.

by a factor of two from the standard far-field criterion, $2 l^{2} / \lambda$, which is derived based on a phase consideration. As long as the region of interest is within $l^{2} / \lambda$ of the turbine, the $2 \mathrm{D}$ scattering model should be fairly accurate. Another observation we can make from Figure 4 is that the field predicted by the 3D far-field approximation is higher than the field computed by the exact numerical integral at close range. This implies that the scattered field estimated using the RCS, which is defined based on the far field, will be too high when the observation position is close to the structure. Van Lil et al. observed this effect and proposed the use of an equivalent height instead of the physical height of the tower when computing the scattered field in the near zone [22, 23]. Since their proposed equivalent size is distance dependent, it 


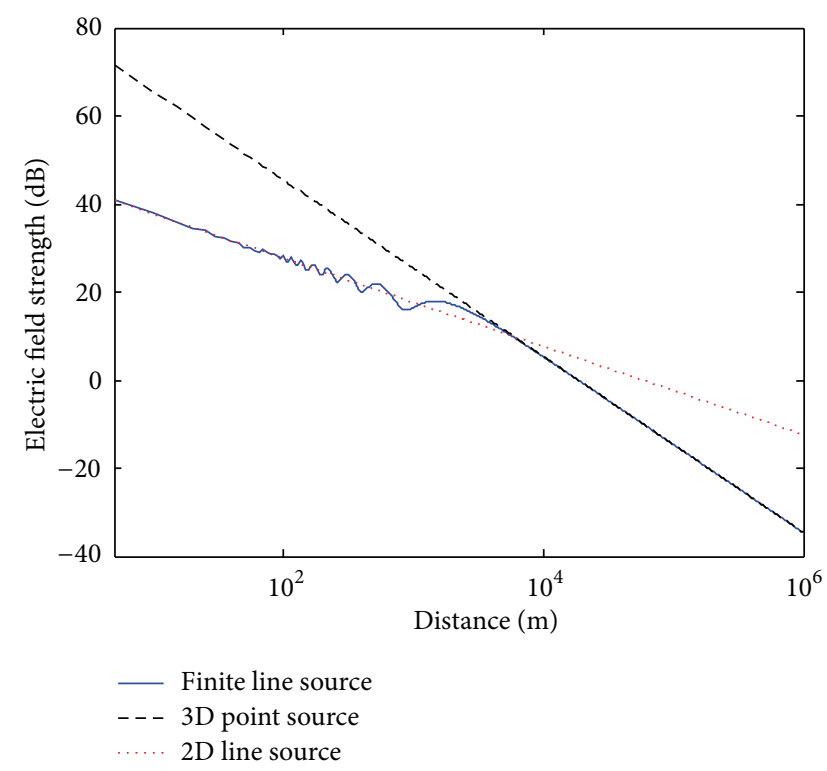

FIGURE 4: Electric field strength versus distance from a finite $64.5 \mathrm{~m}$ line current at $500 \mathrm{MHz}$. The field strengths of an infinite line current and an infinitesimal point current are overlaid, respectively, in red dotted and black dashed lines for comparison.

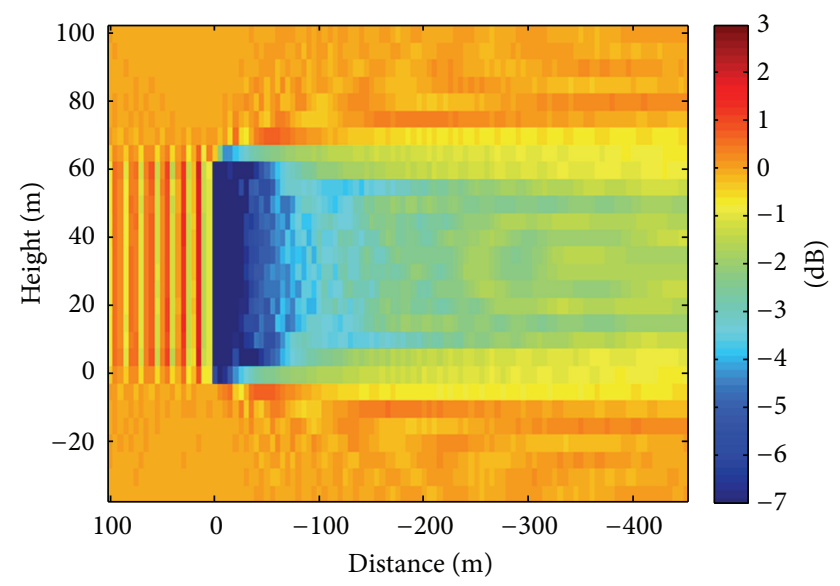

FIGURE 5: Near-field distribution around a 3D finite cylinder in the vertical cut plane along the incident field direction computed using MLFMM. The field inside the shadow region is relatively uniform for different observation heights.

in effect converts the decay of the scattered field from $(1 / r)$ to $(1 / \sqrt{r})$.

So far, the observation height has been restricted at the middle of the tower. Next, we simulate the same 3D finite cylinder using MLFMM and observe the near-field distribution in the vertical cut plane along the incident field direction. The result is shown in Figure 5. Strong shadow boundaries are observed at the bottom and the top edges of the cylinder. This agrees with the optical shadow intuition. More importantly, the field strength is relatively uniform at different observation heights. For example, the RMS

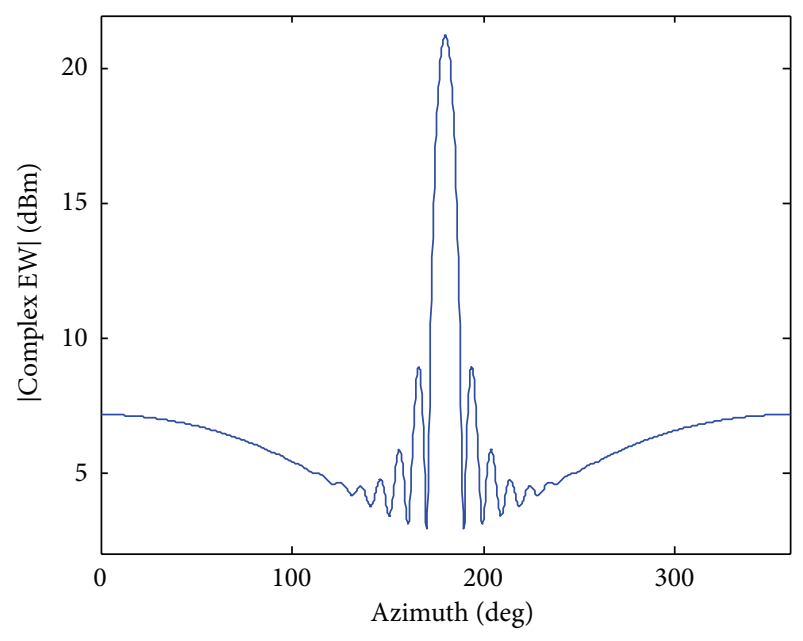

FIGURE 6: Magnitude plot of the complex echo width (EW) of an infinite 2D cylinder.

difference between the horizontal cut at a height of $33.3 \mathrm{~m}$ (Figure 3(a)) and $48.4 \mathrm{~m}$ (not shown) is only $1.39 \%$. This result indicates that the $2 \mathrm{D}$ scattering model can be used to approximate the horizontal cut at various observation heights as long as the observation height stays sufficiently away from the top and the bottom ends of the cylinder.

2.3. Complex Echo Width Approximation. As discussed previously, the near field of a turbine can be readily modeled by a $2 \mathrm{D}$ cylinder. However, computing the near field at many sampling points at $1 \mathrm{GHz}$ and above is still computationally demanded since the computation time scales as the product of the number of observation positions and the number of current basis functions. To further reduce the computation time for the near field, we use the 2D far-field approximation to compute the 2D scattered field. Shown in Figure 6 is the magnitude of the 2D bistatic EW of a 2D circular cylinder with a $3.3 \mathrm{~m}$ diameter simulated via FEKO. The incident azimuth angle is $0^{\circ}$. Although only the magnitude is plotted in Figure 6, the phase front information on the scattered far field is also stored as the phase of the echo width. We then compute the total field by adding the incident plane wave to the scattered field, which is constructed by the complex EW with the proper space decay and phasing as follows:

$$
E^{\mathcal{S}}=E^{i} \frac{e^{-j k r}}{\sqrt{2 \pi r}} \sqrt{\mathrm{EW}} e^{j[\mathrm{phase}(\mathrm{EW})]} .
$$

This approach is similar to that used earlier in [24] for simulating human radar returns. However, a well-defined phase center is assumed for each target part in [24], while no such assumption is needed in the approach presented here. Since the phase of the EW can be regarded as the phase compensation relative to a geometrical reference point, this approach works for all observation directions, including the forward direction. The resulting near-field distribution computed using this approach is shown in Figure 7(b). The same region in Figure 3(b) is copied to Figure 7(a) for 


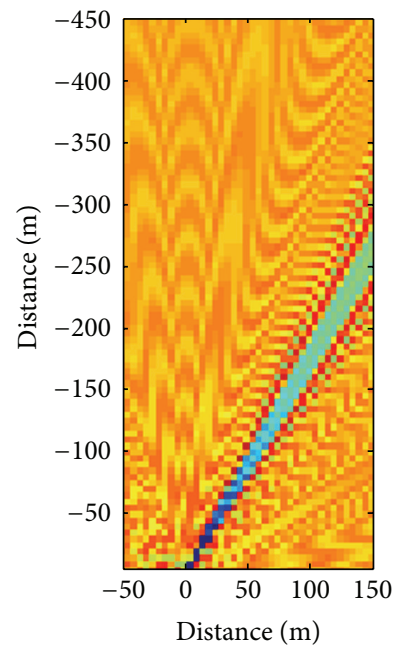

(a) Infinite 2D cylinder

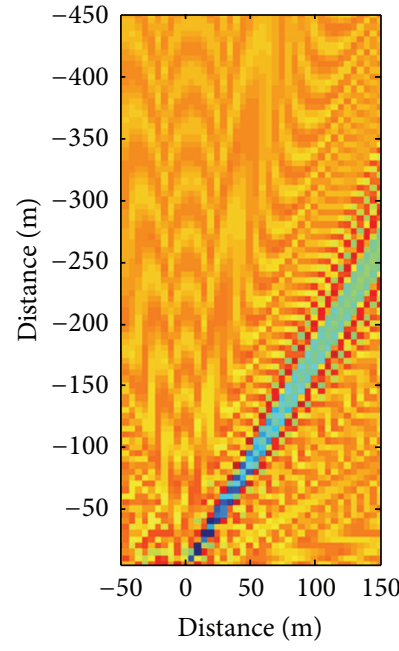

(b) Complex EW approx

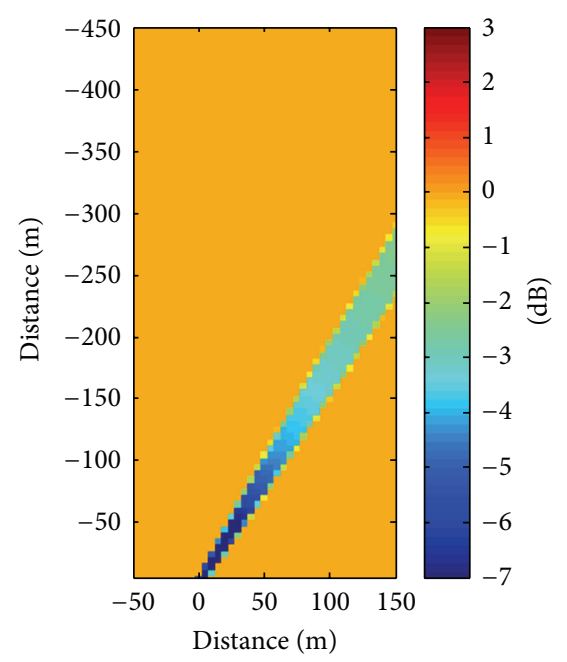

(c) Fresnel approx

FIGURE 7: Computed near-field distribution around an infinite 2D cylinder at $500 \mathrm{MHz}$. (a) Exact numerical integration. (b) Complex echo width (EW) approximation. (c) Fresnel approximation.

comparison. The two results agree well, and the RMS error between the brute-force near-field calculation in Figure 7(a) and the complex EW approximation in Figure 7(b) is $1.36 \%$. Noted that the complex EW approximation requires the distance to be farther than $2 D^{2} / \lambda$, where $D$ is the cylinder diameter. In this case, that distance is $36.5 \mathrm{~m}$, which is quite close to the structure.

For comparison, we also compute the forward shadow using the Fresnel zone blockage formula described in [1113]. The attenuation of the incident field at a position directly behind the turbine is estimated by $1 D / F_{1}$ in linear scale, where $D$ is the diameter of the cylinder and $F_{1}$ is the first Fresnel zone radius, which is given by

$$
F_{1}=\sqrt{\frac{\lambda d_{1} d_{2}}{d_{1}+d_{2}}}
$$

where $\lambda$ is the operating wavelength, $d_{1}$ is the distance from the transmitter to the cylinder, and $d_{2}$ is the distance from the cylinder to the observation position. To extend the previously mentioned formula for an arbitrary position, we solve the geometry problem to find the width of the turbine protruding into the first Fresnel zone, and the protrusion width is used as the diameter $D$ in the blockage formula. It should be noted that the previously mentioned blockage formula will give erratic results when the observation distance is very close since the Fresnel radius can become smaller than the diameter. We use a zero total field when such situation happens. The Fresnel shadow is presented in Figure 7(c). It is observed that the shadow width is properly predicted, but the shadow is too dark. Also, this approach cannot be extended to predict the interference pattern outside the shadow region. The RMS error between Figures 7(a) and 7(c) is 8.24\%.

\section{Multiturbine Results under the Born Approximation and Computation Time}

In the previous section, we established an approximate approach to efficiently compute the field distribution near a single-turbine structure. To extend the approach to a wind farm consisting of tens or hundreds of turbines, we apply the Born approximation and assume each turbine is fully illuminated by the incident field. This approximation is expected to be the least accurate when the turbines are lined up, so that one turbine casts a shadow over subsequent turbines. However, this scenario exists only at very few incident angles, considering the slenderness of the turbine structure and the large spacing between turbines found in offshore wind farms $(600 \mathrm{~m}-1000 \mathrm{~m})$.

To test whether the Born approximation is reasonable, two $64.5 \mathrm{~m}$ long, finite cylinders, each with a $3.3 \mathrm{~m}$ diameter, and spaced $600 \mathrm{~m}$ apart, are simulated rigorously using FEKO's MLFMM solver at $500 \mathrm{MHz}$. The near-field result is plotted in Figure 8(a). The Born approximation in conjunction with the 2D modeling and the complex echo width approximation discussed in the last section are applied to generate the result plotted in Figure 8(b). It is seen that both the shadow region and the interference pattern agree quite well with those in Figure 8(a). The RMS error between Figures $8(\mathrm{a})$ and $8(\mathrm{~b})$ is $3.89 \%$.

Based on the proposed methodology, the near field around a $3 \times 3$ wind farm with a $600 \mathrm{~m}$ spacing between turbines is computed at $150 \mathrm{MHz}, 500 \mathrm{MHz}$, and $3 \mathrm{GHz}$. We use a $2 \mathrm{D}$ cylinder to model each turbine, the complex echo width to compute its near field, and the Born approximation to account for multiple turbines. The results are shown in Figures 9, 10, and 11, respectively. At $150 \mathrm{MHz}$, it can be observed that dark shadow occurs only when the observation position is within $100 \mathrm{~m}$ of the back of the turbine with 


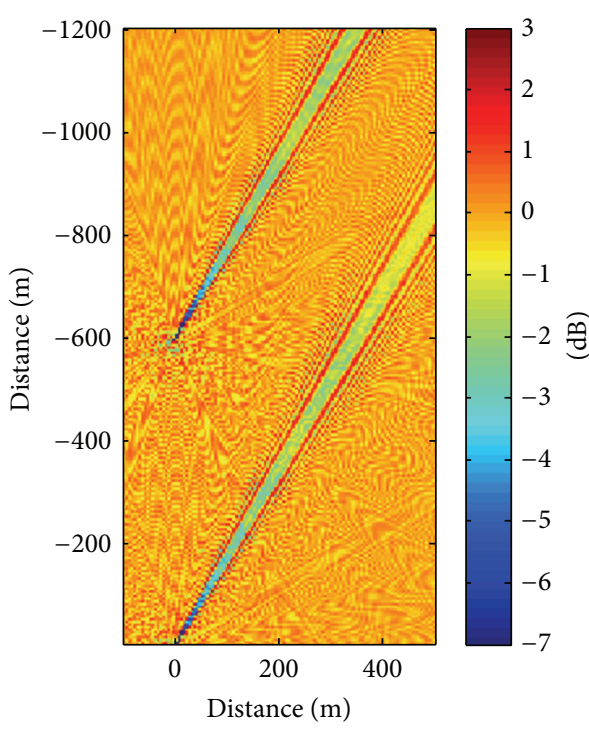

(a) Two finite cylinders

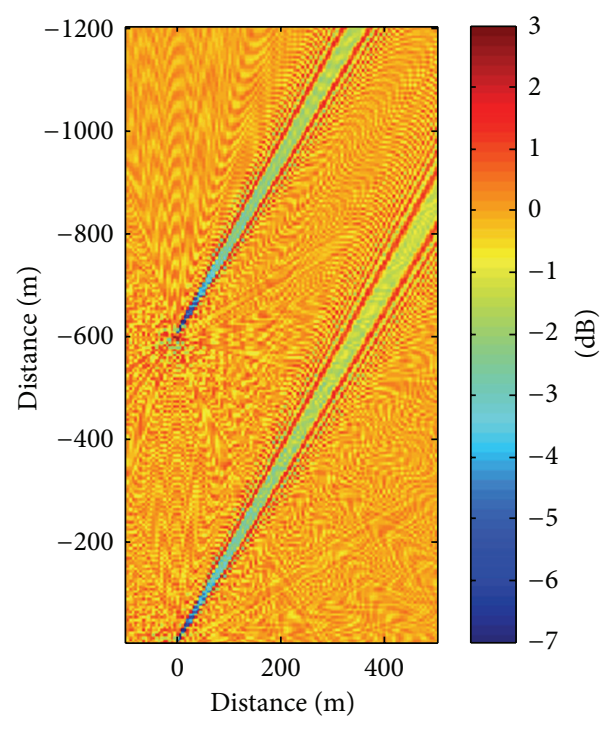

(b) Born approx. + complex EW approx

Figure 8: (a) Near-field distribution around two finite 3D cylinders at $500 \mathrm{MHz}$ computed using the full-wave solver. (b) Near-field distribution around two infinite cylinders generated using the complex EW and the Born approximations.

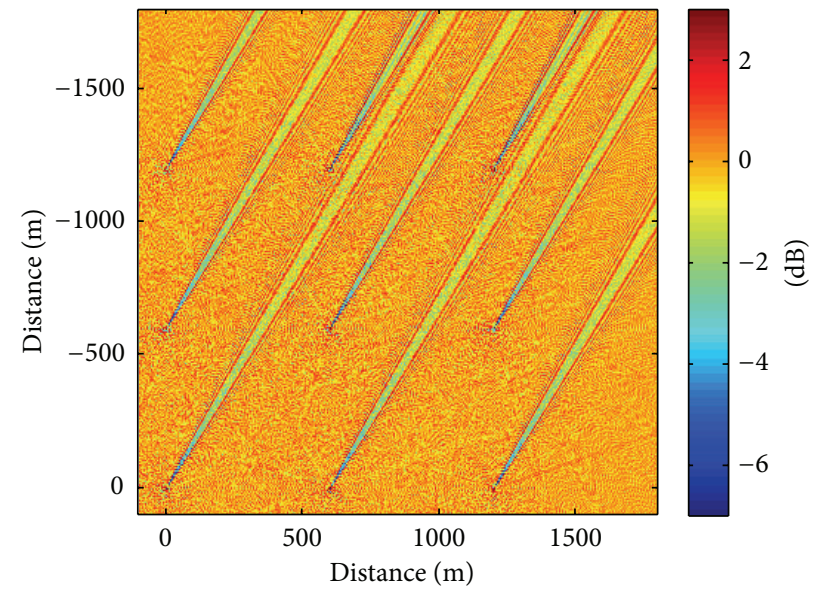

FIGURE 9: Near-field distribution around a $3 \times 3$ wind farm at $150 \mathrm{MHz}$.

respect to the incident direction. The field strength in the shadow region as we move farther away from the turbine becomes almost the same as the field strength outside the shadow region. As the frequency is increased to $500 \mathrm{MHz}$, the shadow becomes darker and extends farther in range. The interference pattern also changes more rapidly as a function of position due to the decrease in wavelength. Figure 11 shows the near-field distribution at $3 \mathrm{GHz}$. It shows a dark and narrow shadow region extending beyond $2 \mathrm{~km}$ behind each turbine. This follows the expected trend since the shadow should eventually approach the geometry optics limit in the very high-frequency limit. The multipath interference pattern also becomes even more rapidly changing as a function of position.

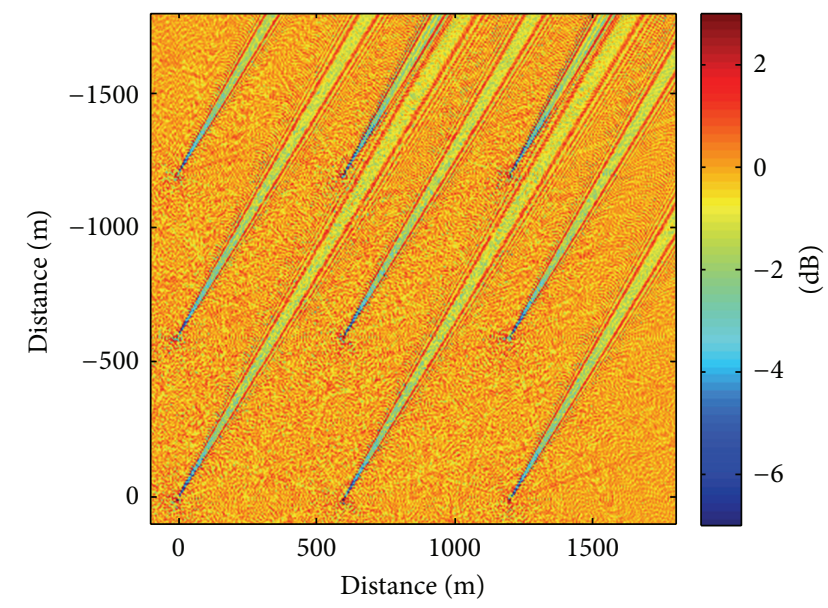

Figure 10: Near-field distribution around a $3 \times 3$ wind farm at $500 \mathrm{MHz}$.

Finally, we discuss the computation time savings from the proposed methodology. The computation time to generate Figures 3(a), 3(b), and 7(b) is summarized in Table 1. All simulations are done on a desktop computer with an Intel Core i7-2600 CPU and $32 \mathrm{~GB}$ of memory. It is noted that the MLFMM utilizes an iterative solver; thus the number of iterations is also included for reference. Comparing the statistics for Figures 3(a) and 3(b), the 2D model reduces the solver time for the induced current by a factor of 779 and reduces the time for the near-field computation by a factor of 10. The overall saving is about a factor of 12. Comparing the statistics for Figures 3(b) and 7(b), the complex EW approach further reduces the time for the near-field computation to a negligible amount. Table 2 summarizes the computation 
TABLE 1: Computation time summary for Figures 3(a), 3(b), and 7(b).

\begin{tabular}{lccc}
\hline & 3D finite cylinder - MLFMM & 2D cylinder - MoM + PBC & 2D cylinder + complex EW \\
\hline Matrix elements & $276 \mathrm{sec}$ & $81 \mathrm{sec}$ & $81 \mathrm{sec}$ \\
Precondition & $171 \mathrm{sec}$ & $0.063 \mathrm{sec}$ & $0.063 \mathrm{sec}$ \\
Solving & $779 \mathrm{sec}(79$ iterations $)$ & $1 \mathrm{sec}$ & $1 \mathrm{sec}$ \\
Near field & $692 \mathrm{sec}$ & $67 \mathrm{sec}$ & $0.027 \mathrm{sec}$ \\
\hline Total time & $31 \mathrm{~min}$ & $2.5 \mathrm{~min}$ & $1.4 \mathrm{~min}$ \\
\hline
\end{tabular}

TABLE 2: Computation time summary for Figures 8(a) and 8(b).

\begin{tabular}{lcc}
\hline & $\begin{array}{c}\text { Two 3D finite } \\
\text { cylinders - MLFMM }\end{array}$ & $\begin{array}{c}\text { Two cylinders - } \\
\text { complex EW + Born }\end{array}$ \\
\hline Matrix elements & $1588 \mathrm{sec}$ & $81 \mathrm{sec}$ \\
Precondition & $185 \mathrm{sec}$ & $0.063 \mathrm{sec}$ \\
Solving & $5905 \mathrm{sec}(100$ & $1 \mathrm{sec}$ \\
Near field & iterations $)$ & $0.132 \mathrm{sec}$ \\
\hline Total time & $77466 \mathrm{sec}$ & $1.4 \mathrm{~min}$ \\
\hline
\end{tabular}

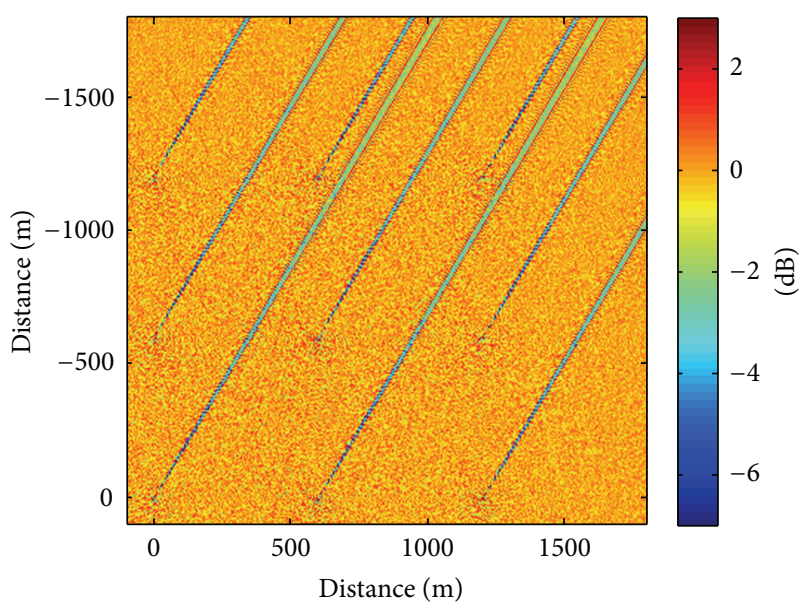

FIGURE 11: Near-field distribution around a $3 \times 3$ wind farm at $3 \mathrm{GHz}$.

time for the two-turbine case shown in Figures 8(a) and 8(b). Comparing the computation time of Figure 8(a) to 3(a), which are both computed using MLFMM, it is noted that the solver time and the near-field computation time are both increased significantly. Lastly, as seen by the time for Figure 8(b), the complex echo width approach reduces the near-field computation time in the two-turbine case from $17466 \mathrm{sec}$ to a negligible amount. Overall, there is a time saving of a factor of 300 between the brute-force approach in Figure 8(a) and the proposed method in Figure 8(b). Such dramatic reduction in computation time means that this method could be quite useful to generate large amounts of data for radio frequency interference assessment.

\section{Conclusions}

In this paper, we have studied the near-field distribution around and within a wind farm under plane wave incidence. To make the problem computationally tractable, the following four assumptions were made. First, we assumed that the electromagnetic scattering is dominated by the tower structure and that the effect of the nacelle and blades is of secondary importance. Second, we assumed that the scattering from the tower can be regarded as a $2 \mathrm{D}$ problem when the observation distance is sufficiently close. Third, the $2 \mathrm{D}$ echo width concept was further applied to simplify the near-field computation. Lastly, we assumed that the interactions between turbines can be neglected and that the Born approximation can be applied when considering a whole wind farm. It was shown through a series of numerical tests that these assumptions can be made without introducing significant errors into the resulting near-field distribution. The near-field distributions of a $3 \times 3$ wind farm were then generated using the proposed methodology at different frequencies. The results showed the spatial extent and depth of the shadows behind the turbine towers as well as the multipath interference between the scattered and incident fields.

It should be noted that the plane wave incidence considered in this paper does not include any phase variations across the scatterers or any realistic decay as a function of distance from the transmitter. Thus, the distance from the transmitter to the wind farm must be sufficiently large to ensure the validity of this assumption. An appropriate modeling methodology for the close-in transmitter case is still needed. It is also important to mention that, while we are initially motivated by offshore wind farms and their impacts on marine radar/communication systems, where both the transmitter and receiver are located along the same plane, changing the elevation angle of the incident field will also be a very interesting study. However, additional formulation will be needed to account for such scenarios. Lastly, when the turbines on a farm are lined up with respect to the incident direction, the Born approximation must be modified to account for the mutual occlusion among the turbines. These will be topics of further study.

\section{Acknowledgments}

This work is supported by the Department of Energy under Grant DE-EE0005380, by the National Science Foundation under Grant ECCS-1232152, and in part by the Texas Norman 
Hackerman Advanced Research Program under Grant no. 003658-0065-2009.

\section{References}

[1] R. J. Vogt, T. D. Crum, W. Greenwood, E. J. Ciardi, and R. G. Guenther, New Criteria for Evaluating Wind Turbine Impacts on NEXRAD Weather Radars, WINDPOWER 2011, Anaheim, Calif, USA, 2011.

[2] Air Warfare Center, The Effects of Wind Turbine Farms on ATC RADARS, Air Warfare Center, Royal Air Force, London, UK, 2005.

[3] Air Warfare Center, Further Evidence of the Effects of Wind Turbine Farms on Air Defense RADARS, Air Warfare Center, Royal Air Force, London, UK, 2005.

[4] "The effect of Windmills on military readiness," United States Department of Defense Report To the Congressional Defense Committees, 2006.

[5] "U.S. Coast Guard Assessment of Potential Impacts to Marine Radar as it Relates to Marine Navigation Safety from the Nantucket Sound Wind Facility as Proposed by Cape Wind, LLC, 2009.

[6] QinetiQ, Results of the Electromagnetic Investigations and Assessments of Marine Radar, Communications and Positioning Systems Undertaken at the North Hoyle Wind Farm, QinetiQ and the Maritime and Coastguard Agency, Salisbury, UK, 2004.

[7] D. Vega, C. Frenandez, O. Grande I Angulo et al., "Software tool for the analysis of potential impact of wind farms on radiocommunication services," in Proceedings of the IEEE International Symposium on Broadband Multimedia Systems and Broadcasting (BMSB '11), Erlangen, Germany, 2011.

[8] B. M. Kent, K. C. Hill, A. Buterbaugh et al., "Dynamic radar cross section and radar doppler measurements of commercial general electric windmill power turbines part 1: predicted and measured radar signatures," IEEE Antennas and Propagation Magazine, vol. 50, no. 2, pp. 211-219, 2008.

[9] A. B. Buterbaugh, B. M. Kent, K. C. Hill et al., "Dynamic radar cross section and radar Doppler measurements of commercial General Electric windmill power turbines Part 2-Predicted and Measured Doppler Signatures," in Proceedings of the 2007 Symposium of the Antenna Measurement Technology Association, St. Louis, Mo, USA, 2007.

[10] E. F. Knott, J. F. Shaeffer, and M. T. Tuley, Radar Cross Section: Its Prediction, Measurement and Reduction, Artech House Radar Library, Norwood, Mass, USA, 1985.

[11] European Organisation for the Safety of Air Navigation, "Assessment methodology to determine the impact of wind turbines on ATC surveillance systems," Eurocontrol Document 0.3, European Organisation for the Safety of Air Navigation, Brussels, Belgium, 2007.

[12] J. J. Lemmon, J. E. Carroll, F. H. Sanders, and D. Turner, "Assessment of the effects of wind turbines on air traffic control radars," National Telecommunications and Information Administration Technical Report 08-454, Department of Commerce, Boulder, Colo, USA, 2008.

[13] S. Hawk, "Impact study of 130 offshore wind turbines in Nantucket Sound," FAA Western Service Area Engineering Services AJW-W15B, 2009.

[14] Meridian Energy Corp, Application Documents of Project Hurunui Wind-Appendix N: Compatibility with Radio Services, Meridian Energy Corp, Wellington, New Zealand, 2011.
[15] A. Theil and L. J. Van Ewijk, "Radar performance degradation due to the presence of wind turbines," in Proceedings of the IEEE Radar Conference, pp. 75-80, April 2007.

[16] G. Greving and M. Malkomes, "Weather radar and wind turbines-an update of the theoretical and numerical analysis of effects," in Proceedings of the 6th European Conference in Meteorology and Hydrology, pp. 1-5, 2010.

[17] S. Jordan, "Test report for evaluation of wind turbines impact on long-range radar surveillance at King Mountain, Texas," Radar Evaluation Squadron Report 84th, Hill Air Force Base, North Davis, Utah, USA, 2008.

[18] A. Frye, "Theoretical and measurement results of the effects of wind turbines of military radar systems and technical assessment methods," in Proceedings of the 60th IEA Topical Expert Meeting, Senternovem, The Netherlands, November 2009.

[19] EM Software \& Systems, FEKO Version 6. 2, EM Software \& Systems Inc., Stellenbosch, South Africa, 2012.

[20] I. Angulo, D. De La Vega, O. Rodríguez, O. Grande, D. Guerra, and P. Angueira, "Analysis of the mast contribution to the scattering pattern of wind turbines in the UHF band," in Proceedings of the 5th European Conference on Antennas and Propagation (EuCAP '11), pp. 707-711, Rome, Italy, April 2011.

[21] C. A. Balanis, Advanced Engineering Electromagnetics, 2/e, John Wiley \& Sons, Hoboken, NJ, USA, 1989.

[22] E. Van Lil, D. Trappeniers, J. W. De Bleser, and A. Van De Capelle, "Computations of radar returns of wind turbines," in Proceedings of the 3rd European Conference on Antennas and Propagation (EuCAP '09), pp. 3852-3856, Berlin, Germany, March 2009.

[23] E. Van Lil, J. W. De Bleser, and A. Van De Capelle, "Computations of the effects of wind turbines in the close near field of RF installations," in Proceedings of the 5th European Conference on Antennas and Propagation (EuCAP '11), pp. 1362-1366, Rome, Italy, April 2011.

[24] S. S. Ram, C. Christianson, Y. Kim, and H. Ling, "Simulation and analysis of human micro-Dopplers in through-wall environments," IEEE Transactions on Geoscience and Remote Sensing, vol. 48, no. 4, pp. 2015-2023, 2010. 

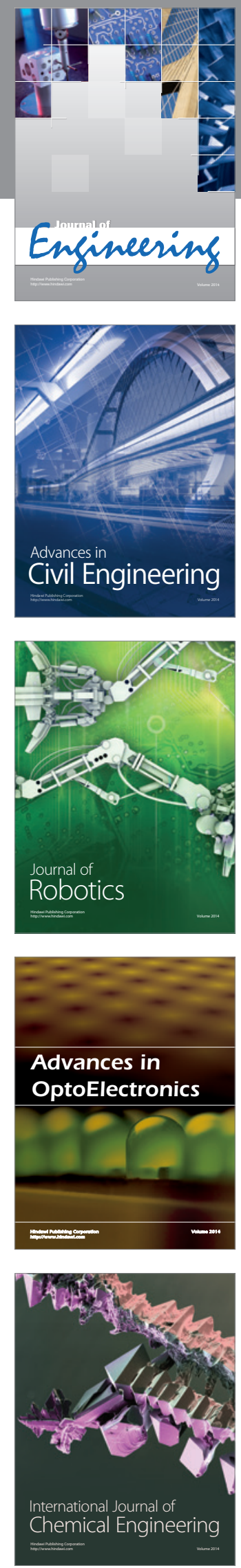

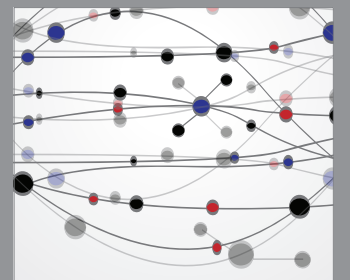

The Scientific World Journal
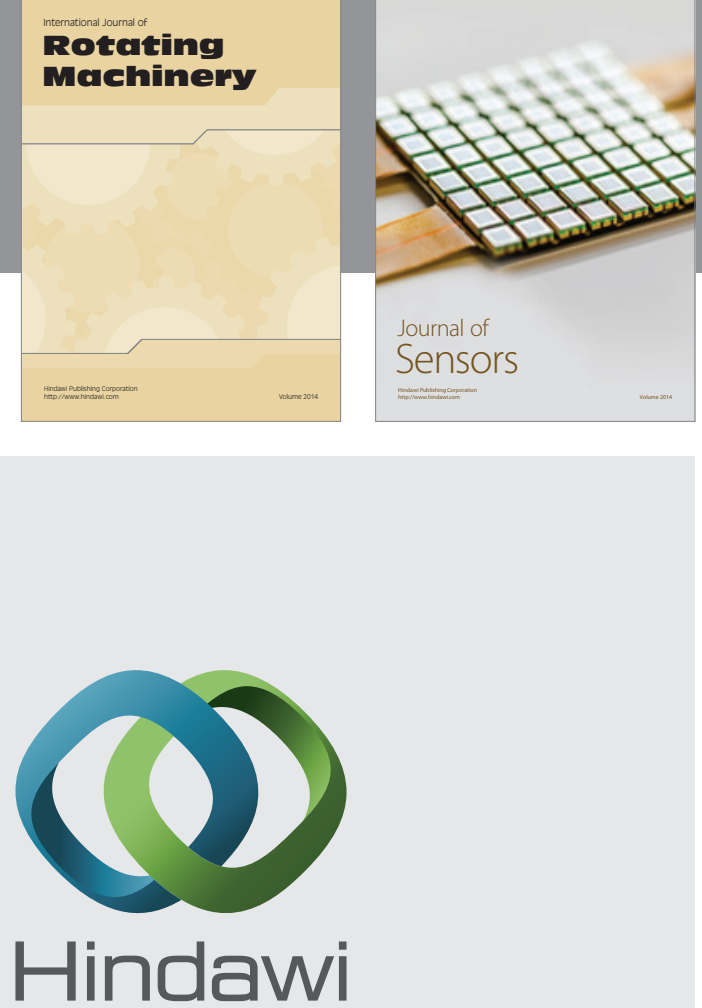

Submit your manuscripts at http://www.hindawi.com
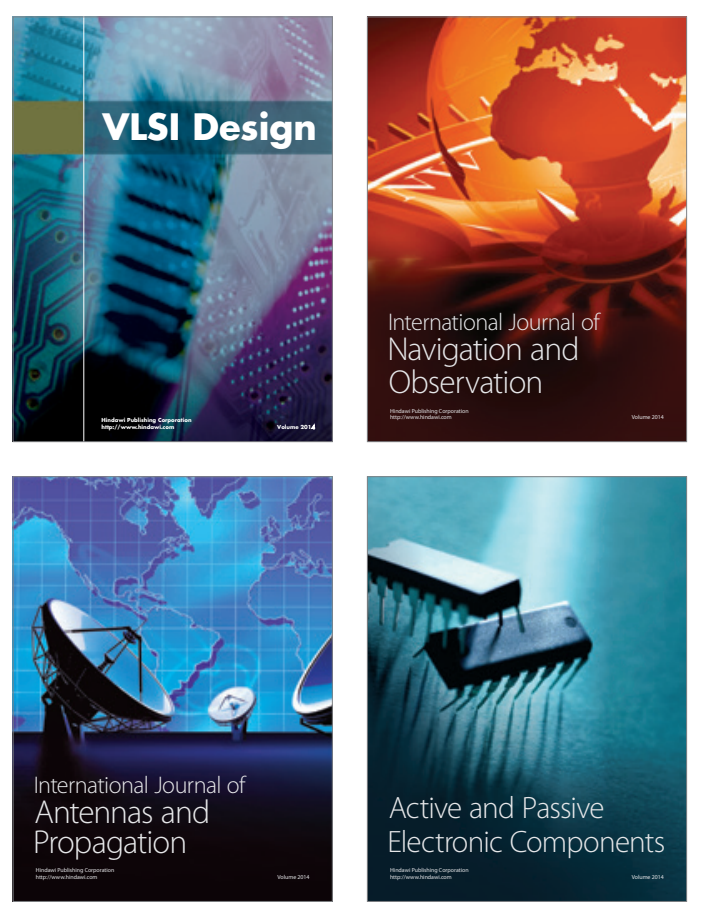
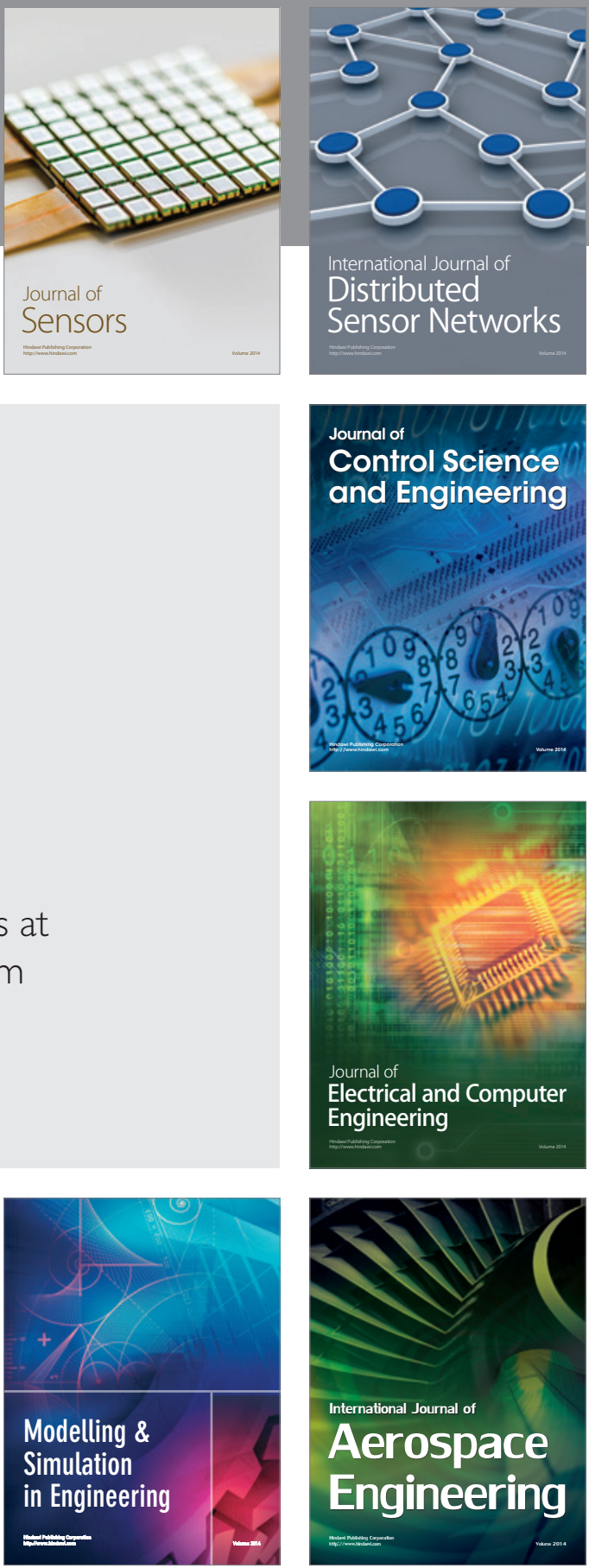

Journal of

Control Science

and Engineering
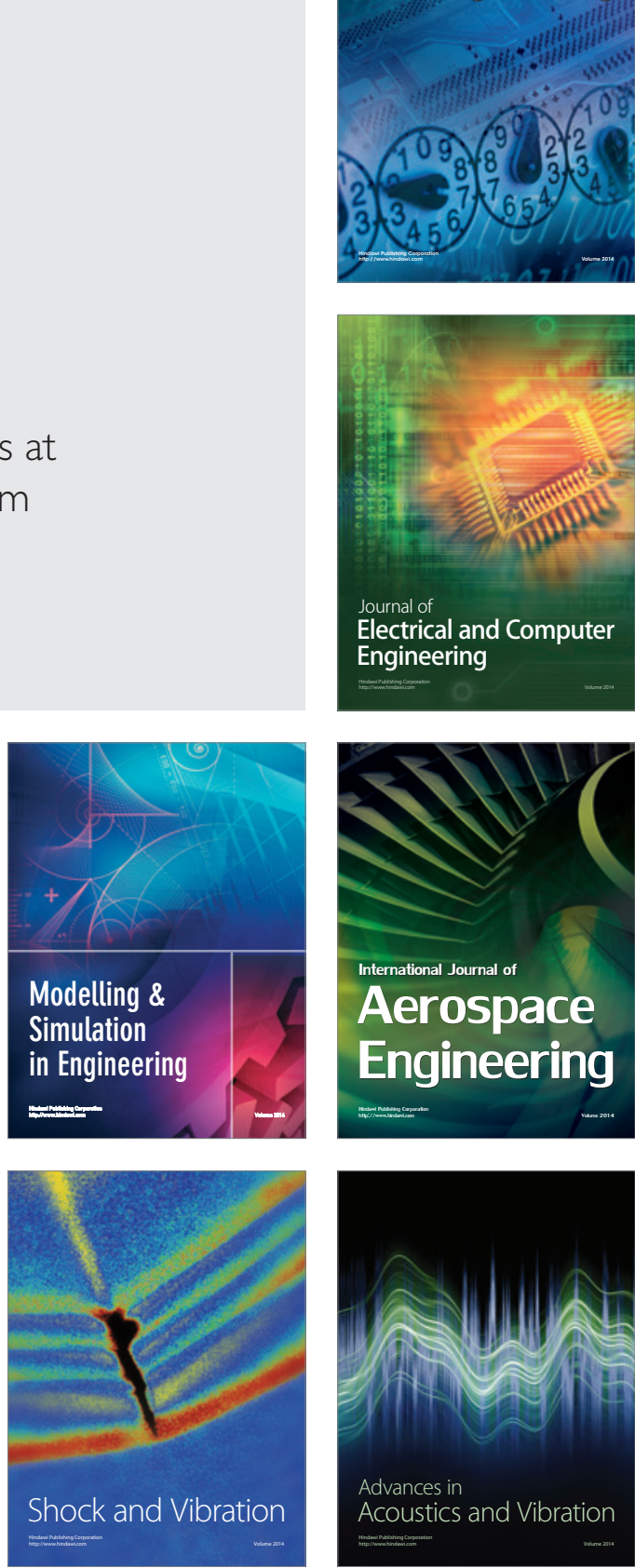